\title{
MDCT FINDINGS IN IATROGENIC DUODENAL PERFORATION
}

Nittala Pramod Philip, Subhash Singla, Rajesh Chakravarthi.

1. Associate Professor. Department of Radiodiagnosis, Christiana Medical College \& Hospital, Ludhiana.

2. Associate Professor. Department of Radiodiagnosis, Christiana Medical College \& Hospital, Ludhiana.

3. Professor. Department of Radiodiagnosis, Christiana Medical College \& Hospital, Ludhiana.

\section{CORRESPONDING AUTHOR:}

Dr. Nittala Pramod Philip.

Department of Radiodiagnosis,

CMC Hospital, Ludhiyana.

E-mail: pramodnittala@yahoo.co.in

ABSTRACT: Iatrogenic duodenal perforation following ERCP/ endoscopic sphincterotomy is a rare complication. Prompt diagnosis and management will help reduce the morbidity and mortality associated with this condition. CT is the imaging modality of choice in patients suspected with duodenal perforation. We present the CT findings in two patients who had iatrogenic duodenal injury.

KEYWORDS: Iatrogenic duodenal perforation, perinephric collection, endoscopic retrograde cholangio-pancreatico-graphy (ERCP), endoscopic sphincterotomy, multi detector computed tomography (MDCT)

\section{Case 1:}

A 45 year old woman had presented with jaundice and upper abdominal pain. Ultrasonography showed cholelithiasis and a dilated CBD. Patient had a duodenal injury while undergoing an ERCP procedure. An erect chest radiograph (CXR) shows dilated bowel loops with no evidence of pneumo-peritoneum (fig 1). Patient was managed conservatively. As the patient developed fever, a CT scan was done which revealed a heterogeneous retroperitoneal collection with multiple air pockets within it. The collection is located in a right perinephric location and is seen to tract along the right psoas muscle (fig 2).

\section{Case 2:}

A 65 year old woman who had undergone cholecystectomy earlier presented with history of jaundice. An MRCP done revealed a mid CBD stricture with dilated intrahepatic biliary radicals (IHBR's). An ERCP was planned to stent the CBD. There was a duodenal injury while negotiating the guidewire. The procedure was abandoned and an immediate chest radiograph done did not reveal air under the diaphragm (fig 1). A CT done shows a right perinephric retroperitoneal collection with few air pockets within it (fig 3).

Imaging Protocol: CT imaging was done on a 128 slice Philips ingenuity CT scanner. CT scan of the abdomen and pelvis was done for both patients with administration of oral contrast for the first patient. Dual phase scanning was done with arterial phase at a delay of $30 \mathrm{sec}$ and the portal venous phase at a delay of $75 \mathrm{sec}$ from the time of injection. $120 \mathrm{ml}$ of non iodinated contrast media was injected at a flow rate of $3 \mathrm{ml} / \mathrm{sec}$ through a power injector. CT sections were taken at a slice thickness of $2 \mathrm{~mm}$ and reconstructed at an interval of $1 \mathrm{~mm}$.

\section{DISCUSSION:}


ANATOMY: The duodenum is an important part of the small bowel as it is in close proximity with the liver, pancreas, gall bladder and the biliary system and diseases affecting these structures may involve the duodenum. The duodenum is the widest part of the small bowel; it has got no mesentery and has both peritoneal and retroperitoneal components. It is approximately $20-25 \mathrm{~cm}$ long and is divided into four parts. The first part or the superior segment extends from the pylorus to the neck of gall bladder. The second part or the descending segment extends from the neck of gall bladder to the genu, at the level of L4 vertebral body. Iatrogenic injuries occur commonly at this level as the ampulla is situated in this segment. The third part or horizontal segment extends from the $4^{\text {th }}$ lumbar vertebra to the level of aorta. This segment is most affected by blunt trauma. The fourth part or the ascending segment extends from the aorta to the ligament of trietz or the duodeno-jejunal junction. ${ }^{1}$

The most severe complications of ERCP are pancreatitis, hemorrhage and perforation. ${ }^{2}$ Duodenal perforation is an acute event and is commonly caused by peptic ulcer disease, endoscopic complication of ERCP or sphincterotomy, and blunt trauma abdomen. ${ }^{3}$

Iatrogenic duodenal perforation is a rare complications occurring in less than $1 \%$ of patients undergoing endoscopic procedures and is usually suspected at the time of endoscopic examination. ${ }^{2}$ Perforation may be due to rupture from the endoscope itself, guidewire puncturing a friable duodenal wall or due to an extended sphincterotomy.

\section{Imaging in suspected duodenal perforations:}

Abdominal and erect chest radiographs have limited value in duodenal perforations as the retroperitoneal air is masked by the overlying bowel loops. There is no free air under the diaphragm as seen in rest of GI tract perforations as the second part of duodenum is situated retro-peritoneal. Sonography also has a limited value in the assessing the retroperitoneal region, especially immediately after the endoscopic procedure when there is lot of bowel distension.

The imaging modality of choice is CT. Multi-detector CT (MDCT) scores in that data is acquired in isotropic voxels and coronal and sagittal reconstruction can be made without compromise in the spatial resolution. CT findings of duodenal perforation include extraluminal air, segmental duodenal wall thickening, periduodenal fat stranding, collection of extraluminal air bubbles in the right perirenal space, focal defect in the bowel wall with oral contrast extravasation and retroperitoneal abscess. ${ }^{3}$ Hainaux et al reported that multi-detector CT has an $86 \%$ predictive value to determine the site of GI tract perforation. ${ }^{4}$

A study by Yagen et al, to determine the extraperitoneal distribution of air following duodenal perforation found that the most common locations are the right anterior pararenal space, right perirenal space and the right extraperitoneal pelvis. ${ }^{3}$ They concluded that duodenal perforation might result in a direct communication of the anterior pararenal space and the right perirenal space.

TREATMENT: Traditionally patients with iatrogenic duodenal perforations have been managed surgically. The indications for surgery include signs of peritonitis, persistent pain or of large perforation involving the lateral duodenal wall. ${ }^{1}$ More recently the trend has been to manage these patients non operatively. Endoscopic treatment of smaller perforation less then $10 \mathrm{~mm}$ in a stable patient with endoclipping devices has gained prominence, especially when detected early. ${ }^{5}$ 
CONCLUSION: Duodenal injuries though uncommon may not present with typical imaging features of a hollow viscus injury like air under the diaphragm on an erect chest x-ray. Since the duodenum is a retroperitoneal structure, air and fluid tend to collect in a retroperitoneal location. Hence in suspected duodenal perforation, CT is the imaging modality of choice and collection in a right perinephric location confirms the diagnosis.

\section{BIBLIOGRAPHY:}

1. Jayaraman MV, Mayo-Smith WW, Morson JS et al. CT of the duodenum: an overlooked segment gets its due. RadioGraphics 2001;21:5147-5160

2. Katsinelos P, Paroutoglou G, Papaziogas B et al. Treatment of a duodenal perforation secondary to an endoscopic sphincterotomy with clips. World J Gastroenterol 2005;11(39):6232-6234

3. Yagen N, Auh YH, Fisher A. Extension of air into the right perirenal space after duodenal perforation: CT Findings. Radiology 2009;250(3):740-748.

4. Hainaux B, Agneesseus E, Bertinotti R et al. Accuracy of MDCT in predicting site of a gastrointestinal tract perforation. AJR Am J Roentgenol 2006;187:1179-1183.

5. Solomon M, Schlachterman A, Morgenstern R. Iatrogenic duodenal perforation treated with endoscopic placement of metallic clips: a case report. Case reports in Medicine vol 2012, article ID 609750. DOI:10.1155/2012/609750.

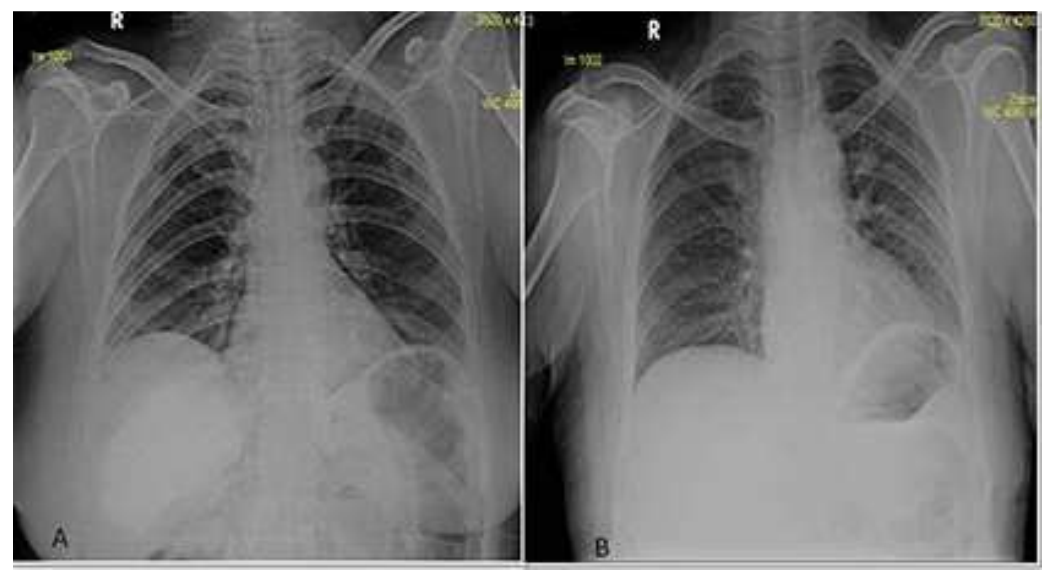

Fig-1

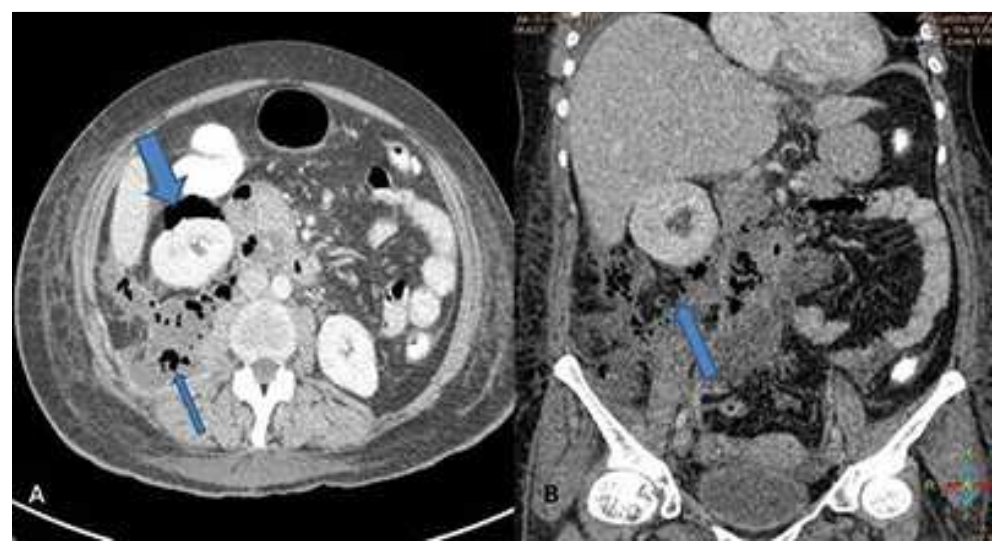

Fig-2 


\section{CASE REPORT}

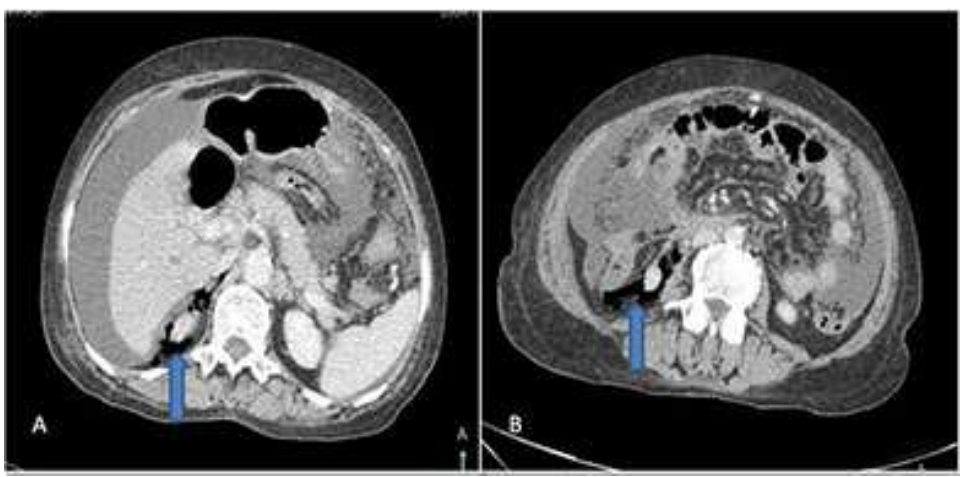

Fig-3 